\title{
Responses of Human Mechanoreceptive Afferents to Embossed Dot Arrays Scanned across Fingerpad Skin
}

\author{
J. R. Phillips, ${ }^{1}$ R. S. Johansson, ${ }^{2}$ and K. O. Johnson ${ }^{3}$ \\ 'University Laboratory of Physiology, Oxford OX1 3PT, United Kingdom, ${ }^{2}$ Department of Physiology, University of Umea, \\ S-901 87 Umea, Sweden, and ${ }^{3}$ Department of Neuroscience, The Johns Hopkins University School of Medicine, \\ Baltimore, Maryland 21205
}

The spatial resolving capacities of the four classes of mechanoreceptive afferents innervating human fingerpad skin were investigated to determine which class sets the limit of tactile spatial resolution for scanning stimuli. The stimulus consisted of an array of embossed dots $(0.7 \mathrm{~mm}$ diameter, $0.5 \mathrm{~mm}$ high) arranged in a tetragonal pattern with dot spacing decreasing linearly from $6.4 \mathrm{~mm}$ at one end of the array to $0.87 \mathrm{~mm}$ at the other. The pattern was wrapped around a drum and repeatedly scanned across the receptive field of single afferents by continuously rotating the drum. Responses to many closely spaced scans were obtained by imposing a lateral shift of the pattern between each revolution. Impulses were recorded microneurographically. Responses were plotted in raster form to produce a neural image of the pattern. Responses of rapidly and slowly adapting type I (FAI and SAI) afferents resolved dots down to a spacing of about $1.5 \mathrm{~mm}$. Responses of type II (FAll and SAII) afferents resolved dots down to a spacing of about 3.5 $\mathrm{mm}$. Variation in scanning speed (range, 20-90 mm/sec) and contact force (range, 0.4-1.0 N) had minimal effects on spatial resolution of all afferents. The response clusters associated with individual widely spaced dots were used to investigate receptive field structure. FAI and SAI fields (mean areas, 6.1 and $4.8 \mathrm{~mm}^{2}$, respectively) each contained several zones of maximal sensitivity. FAl fields had five to eight such zones, whereas SAl fields had three to five such zones. As dot spacing decreased, neighboring dots interacted to affect the responses associated with the individual zones within a field. Initially, one or more zones were deactivated, effectively reducing receptive field size and allowing representation of finer spatial detail than would be predicted from the overall area of the receptive field. At very close dot spacings responses were only obtained when more than one sensitive zone within a field were simultaneously activated by different dots.

\footnotetext{
Received July 23, 1991; revised Oct. 9, 1991; accepted Oct. 10, 1991.

Expcriments were conducted in the Department of Physiology, University of Umea. The study was supported by the Swedish Medical Research Council (Project 14X-08667 and Visiting Scientist Fellowship Project 14V-7702-01), by NIH Grant NS18787, by Wellcome Trust Grant $17733 / 1.27$, and by the University of Umea. We thank L. Backstrom, A. Backstrom, and S. S. Hsiao for technical assistance.

Correspondence should be addressed to Dr. John R. Phillips, University Laboratory of Physiology, Parks Road, Oxford, OX1 3PT, United Kingdom.

Copyright (C) 1992 Society for Neuroscience $0270-6474 / 92 / 120827-13 \$ 05.00 / 0$
}

David Katz claimed that movement is as indispensable for the sense of touch as light is for the sense of vision (Katz, 1925). It is common experience that when the fingers are held stationary on a textured surface its fine spatial details are difficult to appreciate. However, when the skin is scanned laterally across the surface, minute details of the topography are readily discriminated (Katz, 1925; Gordon and Cooper, 1975; Johansson and LaMotte, 1983; Lamb, 1983a; Morley et al., 1983). Katz attributed the marked difference between stationary and scanned touch to the fact that movement recruits the "vibration sense" that may have a higher acuity than the "pressure sense" of stationary touch. Similar but more explicit mechanisms have been proposed (Johnson and Lamb, 1981; Johnson and Phillips, 1984; Srinivasan and LaMotte, 1987), namely, that (1) movement prevents adaptation in mechanoreceptor responses generally, resulting in an enhanced spatial representation of a moving surface compared to a stationary surface; (2) movement recruits rapidly adapting (RA) as well as slowly adapting (SA) afferents so that a moving surface is sampled more densely than a stationary surface; and (3) movement allows the transmission of spatial information about a surface in the form of temporal modulation of afferent responses.

In order to investigate the enhanced acuity of scanning versus static touch, and before possible spatial mechanisms (e.g., mechanisms 1 and 2 above) can be differentiated from nonspatial mechanisms (e.g., mechanism 3 above), a description of the responses of human mechanoreceptive afferents to moving stimuli is required. However, most studies of human afferents have employed simple, stationary (punctate or edge) stimuli (Johansson, 1978; Johansson et al., 1982). Description of their responses to moving, spatially distributed stimuli is confined to one limited study of responses of human afferents to Braille characters (Phillips et al., 1990). There have been several studies of the responses of monkcy afferents to spatially complex stimuli, including stationary gratings and edges (Phillips and Johnson, 1981 a; Srinivasan and LaMotte, 1987) and moving gratings, edges, and dot patterns (Darian-Smith and Oke, 1980; Darian-Smith et al., 1980; Johnson and Lamb, 1981; Lamb, 1983b; LaMotte and Whitehouse, 1986; Goodwin and Morley, 1987a,b; LaMotte and Srinivasan, 1987a,b; Morley and Goodwin, 1987; Goodwin et al., 1989; Sathian et al., 1989; Connor et al., 1990). The studies of monkey afferents indicate that the SA population probably conveys the most faithful information about the spatial structure of a surface (Darian-Smith et al., 1980; Johnson and Lamb, 1981; Phillips and Johnson, 1981a; Srinivasan and LaMotte, 1987) whereas the RA population may 


\section{Location along pattern $(\mathrm{mm})$}
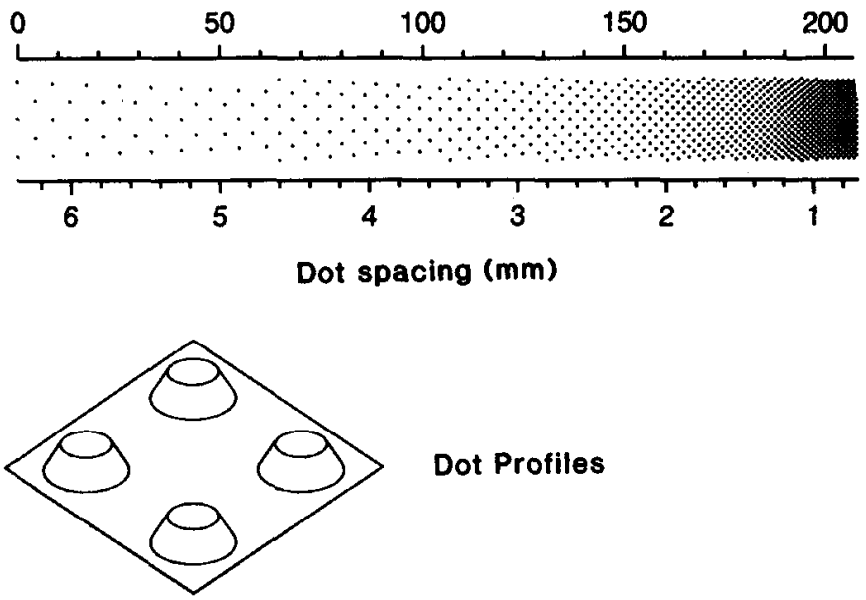

Dot Profiles

Figure 1. Tetragonal array of embossed dots used as the stimulus. The array, shown laid out flat at top, was formed on a flexible nylon plate that was wrapped around the circumference of a $67-$ inm-diameter drum. Center-to-center dot spacing decreased linearly from 6.4 to $0.87 \mathrm{~mm}$ (left to right, lower scale) with the exception of a $10 \mathrm{~mm}$ patch at the right within which dot spacing remained constant at $0.87 \mathrm{~mm}$. The upper scale shows circumferential distance from the joint where the two ends of the pattern met when wrapped around the drum. Each embossed dot had the form of a truncated cone (shown at bottom) with a $0.7-\mathrm{mm}$ diameter flat top, a height of $0.5 \mathrm{~mm}$, and a base diameter of approximately $1.0 \mathrm{~mm}$.

convey intensive information relating to the height of dots and sharpness (or shape) of edges (LaMotte and Whitehouse, 1986; Srinivasan and LaMotte, 1987). In order to extrapolate results from monkey to man it must be assumed that the peripheral neural mechanisms (including skin mechanics) are the same in both species. Four classes of mechanoreceptive afferents, SA types I and II (SAI and SAII) and RA types I and II (FAI and FAII) are reported for human glabrous skin (Vallbo and Johansson, 1984). However, the occurrence of SAII afferents has not been reported in studies of monkey glabrous skin. Moreover, though it is generally assumed that the response properties (e.g., receptive field sizes) of SAI, FAI, and FAII afferents in human are similar to SA, RA, and PC (Pacinian) afferents in monkey, no quantitative comparison has been made.

This study describes the responses of human mechanoreceptive afferents to scanning dot patterns and compares the spatial resolving capacities of the four afferent classes. The objective is to determine which class sets the critical dimension below which spatial information is not represented within the spatial pattern of response in peripheral afferents. This dimension sets a lower limit on central mechanisms that resolve spatial detail of a stimulus based on the spatial patterning of peripheral responses (i.e., tactile spatial resolution). It does not set a limit on nonspatial mechanisms (e.g., those that might extract spatial information from temporal modulation of peripheral responses).

\section{Materials and Methods}

Human subjects. Experiments were conducted on 17 adults ( 10 females, 7 males) aged 20-33 years. The informed consent of all subjects was obtained according to the declaration of Helsinki. Subjects sat in a dentist's chair with the right upper arm slightly abducted and the forearm extended anterolaterally to rest in a vacuum cast fixed to a table. The hand was embedded palm up in modeling clay, leaving the volar aspect accessible for stimulation. Finger clamps were occasionally used for additional fixation. Impulses were recorded from single tactile af- ferents with tungsten needle electrodes inserted percutaneously into the median nerve, $5-10 \mathrm{~cm}$ proximal to the elbow (Vallbo and Hagbarth, 1968).

Fiber classification. Afferents were classified in accordance with criteria described elsewhere (Talbot et al., 1968; Knibestol and Vallbo, 1970; Johansson and Vallbo, 1979). Briefly, the initial distinction between rapidly and slowly adapting afferents was made on the basis of the response to a steadily maintained skin indentation. Afferents responding continuously to a steady indentation were classified as slowly adapting; those responding transiently, that is, only during application and removal of the stimulus, were classified as rapidly adapting. The latter were further subdivided into FAI and FAII on the basis of the size of the receptive field and on absolute sensitivity. Rapidly adapting afferents having small, well-defined receptive fields (about $10 \mathrm{~mm}^{2}$ when mapped with von Frey hairs at four to five times the threshold force) were classified as FAl; those with large, poorly defined receptive fields (typically extending over a whole digit, but often responding to gentle taps over the entire hand) were classified as FAII. In addition, many FAII afferents responded readily to gentle puffs of air directed across the receptive field, whereas FAI afferents did not. Slowly adapting afferents were subdivided into two types on the basis of receptive field size and the response properties at the point of maximum sensitivity as compared with the surround. SAI afferents had small, well-defined receptive fields (about $10 \mathrm{~mm}^{2}$ when mapped with von Frey hairs) containing several points of high sensitivity. SAII afferents had larger receptive fields with indistinct boundaries. In addition, the response at the point of maximum sensitivity in SAII fields was sluggish by comparison with the response evoked at the point of maximum sensitivity in SAI fields. SAII afferents invariably responded to horizontal skin stretch, but this response property was not by itself sufficient to distinguish them from SAI afferents. All single units studied quantitatively had receptive fields on the glabrous skin, with the majority (74\%) located on the distal fingerpad.

Stimulus surface. The stimulus consisted of an array of embossed dots arranged in a tetragonal pattern $25 \mathrm{~mm}$ wide by $212 \mathrm{~mm}$ long, with center-to-center dot spacing decreasing linearly from $6.4 \mathrm{~mm}$ to 0.87 $\mathrm{mm}$ along the major axis of the pattern (Fig. 1). The embossed pattern was produced by exposing a photosensitive nylon polymer (Toyobo Printight) to ultraviolet light through a mask consisting of a photographic negative of the dot pattern. The embossed dots had the form of truncated cones with a $0.7-\mathrm{mm}$-diameter fiat top, a base diameter of $1.0 \mathrm{~mm}$, and a height above the background of $0.5 \mathrm{~mm}$. This stimulus pattern has a number of advantages for the analysis of spatiotemporal response properties. First, the repetitive pattern produces repetitive responses that are easily differentiated from noise and may be averaged for greater definition. Second, the stimulus pattern provides a nearly continuous range of dot spacings so that the resolution limit of a neuron's spatiotemporal response properties can be determined. Third, the twodimensional structure of the tetragonal pattern provides a basis for distinguishing between neuronal responses that are sensitive to the spatial structure of the surface from those that are merely sensitive to the temporal cadence of the stimulus.

Rotating drum stimulator. A rotating drum stimulator (Johnson and Phillips, 1988) was used to deliver the embossed pattern to the skin in a manner simulating the relative motion between the skin and a surface when a finger is scanned across a surface. The embossed pattern was glued around the periphery of a drum ( $212 \mathrm{~mm}$ circumference), which was applied to the skin with a controlled force and rotational velocity. The drum remained in contact with the skin for 50-100 revolutions. Once per revolution the drum was advanced $0.2 \mathrm{~mm}$ along its axis of rotation (i.e., at right angles to the scanning direction) causing the pattern to advance incrementally across the receptive field of the fiber under study on each successive parallel sweep (Johnson and Lamb, 1981). The instantaneous position of the drum was monitored by means of marker pulses (200 per revolution) emitted by a shaft encoder mounted on the drum shaft. Scanning velocity and contact force ranged between 20 and $90 \mathrm{~mm} / \mathrm{sec}$ and 0.4 and $1.0 \mathrm{~N}$, respectively. These velocities and forces lie within the ranges typically used during active scanning of surfaces with the finger tips (Lederman, 1974). Generally, the pattern was scanned across the skin of the fingerpad in the proximal-to-distal direction. During experiments, occurrence times of spikes and drum angular location markers were recorded (to $0.1 \mathrm{msec}$ resolution) using a timing interface and an IBM PC/AT microcomputer, which also displayed the spike rasters as they were collected.

Spatial event plots. The serial stream of action potentials produced 


\section{Location along pattern, $\mathrm{mm}$ (surface movement: $\longleftarrow$ )}
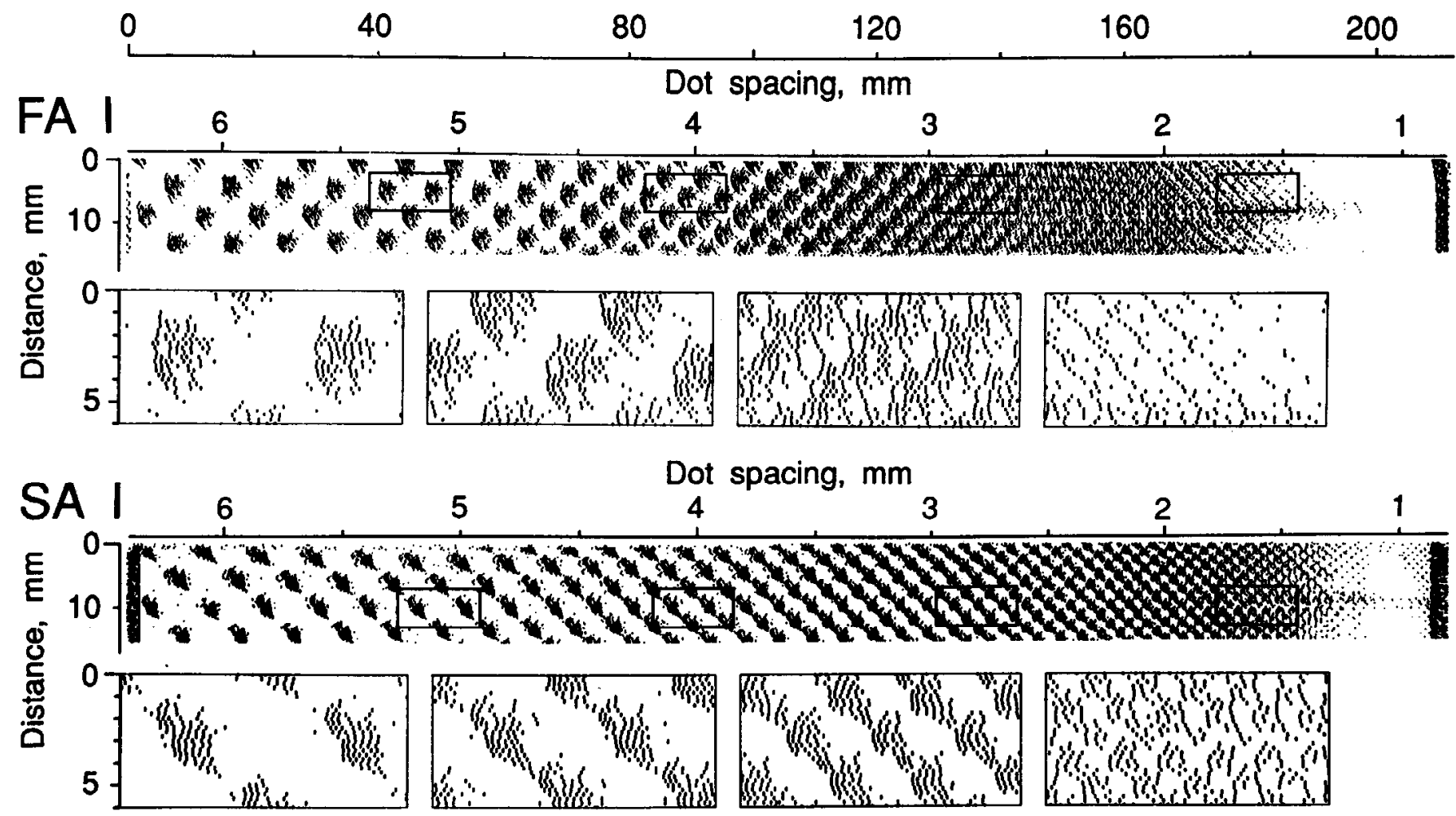

Figure 2. Spatial event plots reconstructed from responses of a typical FAI fiber and a typical SAI fiber. Responses were obtained by repeatedly scanning the tetragonal dot pattern (Fig. 1) across the skin at $60 \mathrm{~mm} / \mathrm{sec}$, with a shift $(0.2 \mathrm{~mm})$ of the stimulus interposed between scans. Application force, $0.6 \mathrm{~N}$. Responses to the whole pattern are shown as the long horizontal response blocks in which each small dot represents the occurrence of one action potential. The top rows in each raster plot represent early scans, while the bottom rows represent late scans. The four sections of each response (small rectangles) shown at higher magnification $(3.5 \times)$ illustrate the repeatability of the responses.

by the stimulation procedure was converted to a two-dimensional spatial event plot (SEP) by laying out the impulse sequences evoked on successive rotations as parallel rows of ticks (see Fig. 2). The location of each action potential was computed relative to the marker pulses emitted by the shaft encoder by an interpolation algorithm with a resultant accuracy of approximately $0.01 \mathrm{~mm}$ (Johnson and Phillips, 1988). The average number of impulses per millimeter of surface movement, the average frequency of action potentials, and the average number of action potentials evoked by each dot were computed as a function of dot spacing. These averages were obtained by analyzing the responses in a "moving window" that extended across the full width of the SEP (i.e., at right angles to the sweep direction) and along the SEP (i.e., along the sweep direction) by an amount equal to three times the local dot spacing. Because of the continuously varying dot spacing in the sweep direction, the dot spacing within the analysis window deviated from the mean value by a small amount ( $4 \%$ maximum).

\section{Results}

Recordings were obtained from $22 \mathrm{FAI}$ fibers (19 receptive fields on the distal and 3 on the middle phalanx), 15 SAI fibers (10 receptive fields on the distal and 5 on the middle phalanx), 8 FAII fibers ( 3 receptive fields on the distal, 4 on the middle, and 1 on the proximal phalanx), and 7 SAII fibers (6 receptive fields on the distal and 1 on the middle phalanx). The responses of a small subset of these fibers to scanned Braille characters have been described in a previous report (Phillips et al., 1990).

\section{FAI and SAI responses to scanned arrays}

SEPs reconstructed from the responses of single representative FAI and SAI afferents are illustrated in Figure 2. The overall response pattern is shown for each fiber together with rectangular sections of the response pattern that have been magnified $(3.5 \times)$ in order to illustrate the structure within the response. Each action potential (dot in Fig. 2) is plotted at a horizontal and vertical position corresponding to the angular and axial location of the drum at the time of the action potential. The responses shown in Figure 2 are representative of the FAI and SAI fiber samples in terms of the clarity of representation of the stimulus pattern; for each fiber type there is a clear representation of the stimulus topography in the response patterns for coarse dot spacing. With decreasing dot spacing the representation of surface detail is (visually) identifiable down to about $1.5 \mathrm{~mm}$ dot spacing. At these close spacings the underlying tetragonal pattern is generally more faithfully represented within the SAI responses than within the FAI responses. At smaller dot spacings the response patterns show less evidence of the surface geometry until the fibers cease firing at about $1 \mathrm{~mm}$ dot spacing. However, even at the finest dot spacings to which FAI and SAI afferents respond, the impulse patterns are not random but appear to be related to the tetragonal arrangement of the dots, implying that both FAI and SAI units are sensitive to the surface detail of this particular pattern whenever they respond. The magnified sections in Figure 2 show that both fiber types have remarkably repcatablc responses; the fine details of the response patterns evoked by adjacent dots are very similar. In addition, there is no detectable long-term adaptation in the responses. Both SEPs in Figure 2 were constructed from re- 
FAI $(n=22)$
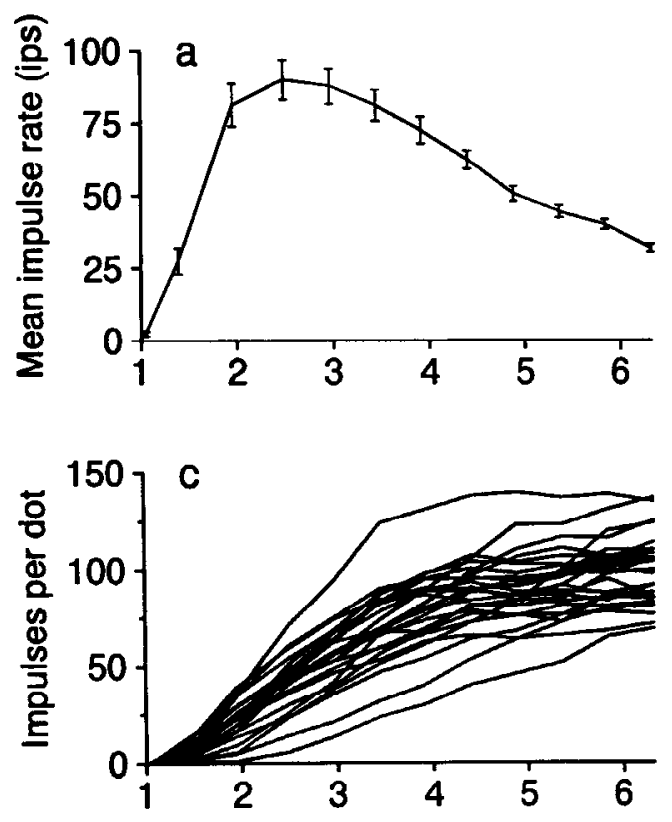

Fean discharge rate and number of impulses evoked per dot versus dot spacing for FAI and SAI fibers. For all data, stimulus scanning velocity was $60 \mathrm{~mm} / \mathrm{sec}$ and application force was 0.6 N. $a$ and $b$, Mean rate $( \pm 1$ SE) versus dot spacing for $22 \mathrm{FAI}$ and $15 \mathrm{SAI}$ afferents; both fiber types respond maximally at a dot spacing of 2.5-3 mm. $c$ and $d$, Number of impulses evoked per dot versus dot spacing for each FAI and SAI fiber. $e$ and $f$, Mean number of impulses per dot $( \pm 1 \mathrm{SE})$ versus dot spacing for data shown in $c$ and $d$. These measures were obtained by averaging within a "moving window" extending across the spatial event plot as described in Materials and Methods.

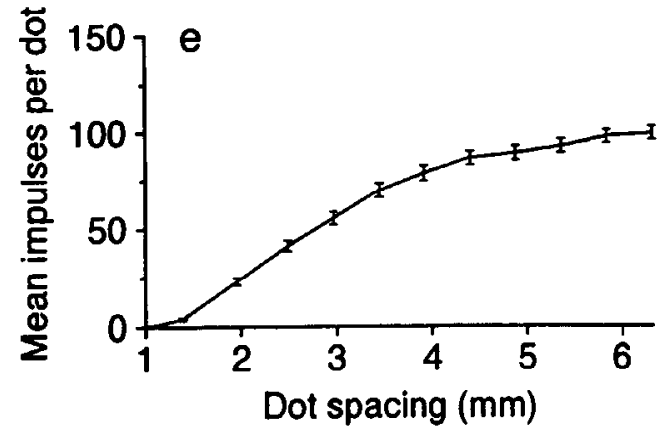

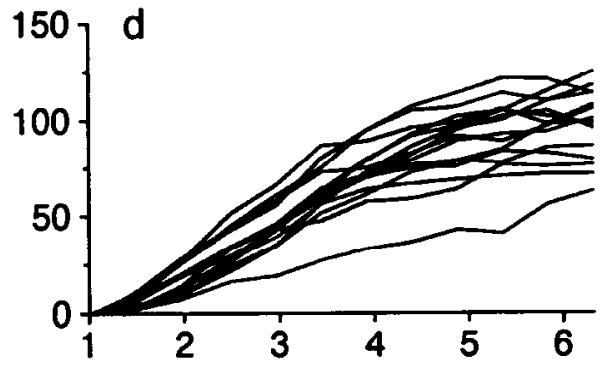

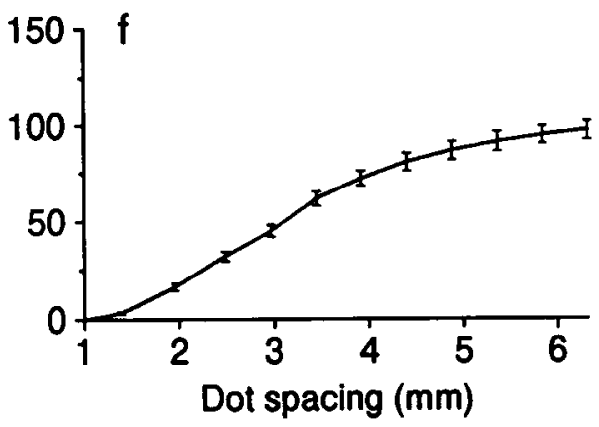

sponses to approximately 4.5 min of continuous stimulation and are composed of many thousands of action potentials, yet in both, the responses to the early and late set of sweeps are essentially the same. Figure $3, a$ and $b$, shows the mean response rates as a function of dot spacing for all FAI and SAI afferents. Mean discharge frequency increases rapidly with increasing dot spacing for both fiber types until a peak is reached at a dot spacing of $2.5 \mathrm{~mm}$ for FAI and $3.0 \mathrm{~mm}$ for SAI afferents. As dot spacing is increased further, mean response rates fall. The spatial event plots of Figure 2 show that at coarse dot spacings each dot of the pattern is represented by a clustcr of impulses. As dot spacing decreases the clusters of impulses converge and coalesce and the number of impulses evoked by each dot declines. The quantitative relationship between dot spacing and the number of impulses evoked by each dot is shown in Figure $3 c-f$ for $\mathrm{FAI}$ and SAI afferents. All behave in a similar manner. As dot spacing increases, the number of impulses per dot increases almost linearly for dot spacings up to about $4.0 \mathrm{~mm}$. Thereafter, the number of impulses per dot increases more slowly; that is, neighboring dots spaced closer together than about $4.0 \mathrm{~mm}$ progressively interact to suppress responses to the individual dots, but beyond about $4.0 \mathrm{~mm}$ this interaction becomes much weaker.

\section{FAII and SAII responses to scanned arrays}

Spatial event plots reconstructed from the responses of single representative FAII and SAII afferents are illustrated in Figure 4. The responses of these afferent types do not resolve the spatial detail of the stimulus as clearly as do the FAI and SAI responses shown in Figure 2. Most FAII and SAII responses showed some visually identifiable representation of the stimulus pattern at dot spacings above $3-4 \mathrm{~mm}$, but below this spacing clear tetragonal patterning (as judged visually) was observed in the responses of only one FAII fiber. The SAII response patterns are qualitatively different from those obtained from the other three afferent types. The widely spaced dots typically evoke one or two impulses per passage of the dot over the receptive field, producing "impulse fronts" in the SEP (Fig. 4, bottom left). As with the SAI and FAI responses, there is no detectable longterm adaptation in either the FAII or SAII responses. Figure 5 shows that dot spacing has little effect on FAII and SAII mean impulse rate. The FAII fibers generally show a rather uniform response rate to all parts of the stimulus pattern, though for the mean of the eight FAII afferents, peak firing rate occurs at a dot spacing of 3-4 mm. Spurious vibration in the stimulator (originating in the motor and gear train used to rotate the drum) 


\section{Location along pattern, mm (surface movement: $\longleftarrow$ )}
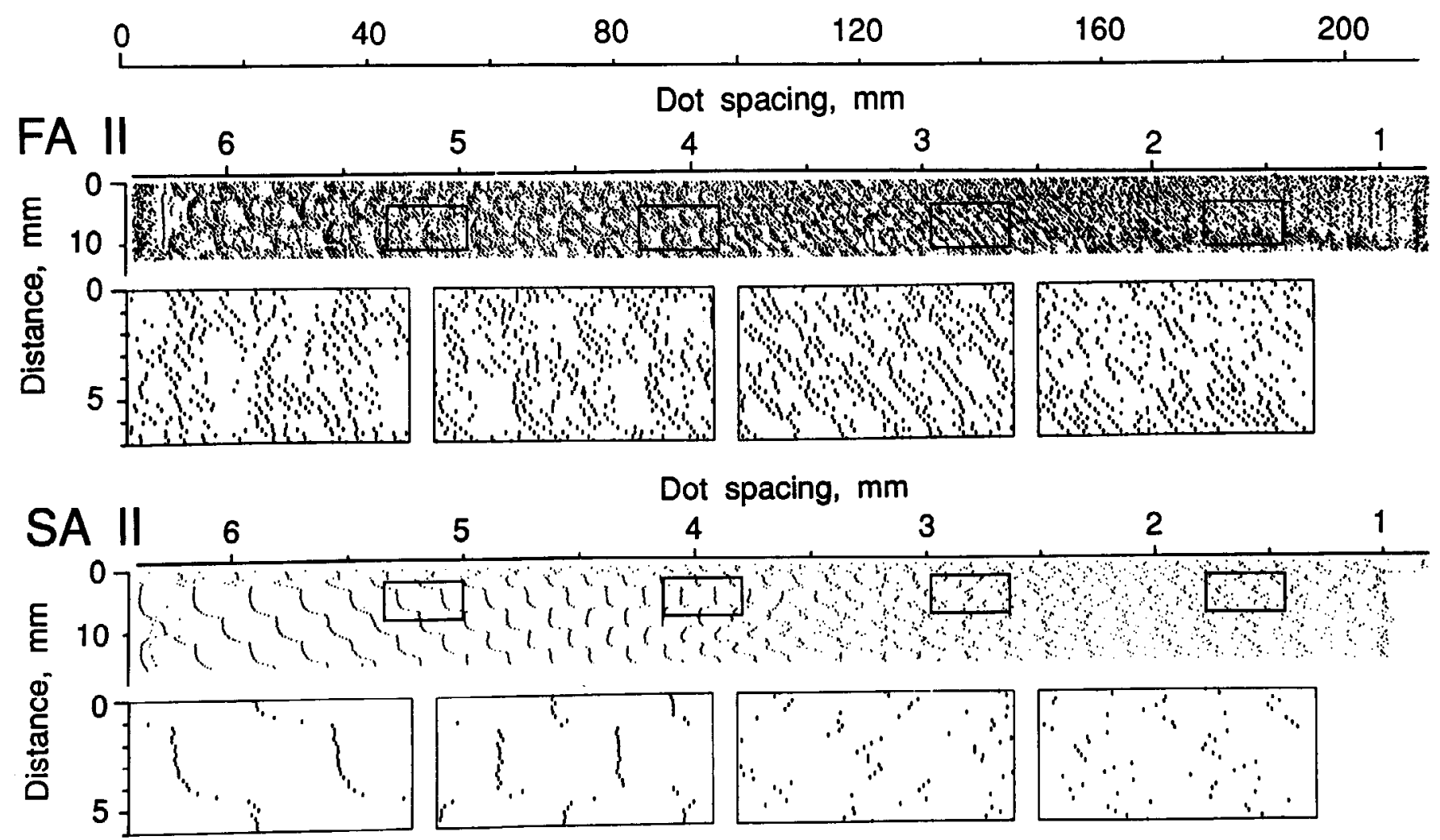

Figure 4. Spatial event plots reconstructed from responses of a typical FAII fiber and a typical SAII fiber. Responses were obtained by repeatedly scanning the tetragonal dot pattern (Fig. 1) across the skin at $60 \mathrm{~mm} / \mathrm{sec}$, with a shift $(0.2 \mathrm{~mm})$ of the stimulus interposed between scans. Application force, 0.6 N. Responses to the whole pattern are shown as the long horizontal response blocks in which each small dot represents the occurrence of one action potential. The top rows in each raster plot represent early scans, while the bottom rows represent late scans. The four sections of each response (small rectangles) shown at higher magnification $(3.5 \times)$ illustrate the repeatability of the responses.

excited these highly sensitive units, and this may have contributed to their uniformity of response. For the SAII afferents the mean impulse rate evoked by the stimulus pattern was remarkably constant at all dot spacings and was almost an order of magnitude lower than for the other three afferent types. Figure 5 shows that the mean number of impulses evoked per dot increascs nearly linearly with increasing dot spacing for all FAII and SAII fibers. This results from the fact that although mean rate remains nearly constant, the density of dots in the pattern decreases as dot spacing increases.

\section{Effects of scanning velocity: FAI and SAI afferents}

The effect of scanning velocity on response was investigated for 13 FAI and 8 SAI afferents. Figure 6 shows sections of SEPs from a representative fiber of each type at three velocities $(20$, 60 , and $90 \mathrm{~mm} / \mathrm{sec}$ ) and four different dot spacings. The most striking effect for both types is a reduction in the spatial density of action potentials with increasing scanning velocity. However, the representation of the spatial detail of the stimulus is hardly affected by variations in scanning velocity within this range. Figure 7 shows the effect of scanning velocity on mean firing rates and numbers of impulses per dot as a function of dot spacing for the FAI and SAI afferents illustrated in Figure 6. The shapes of the curves in Figure 7 are influenced little by scanning velocity. A small effect is a tendency for the fibers to respond to slightly closer dot spacings at lower velocities. In- creases in impulse rate are not proportionate to velocity, which results in a declining relationship between impulses per dot and velocity. In Figure 7 the dot spacing at which the maximum discharge rates occur (about $2.0 \mathrm{~mm}$ for the FAI and $2.5 \mathrm{~mm}$ for the SAI) remains unaffected by scanning velocity, indicating that it is the spatial rather than the temporal characteristics of the stimulus that determine the response patterns in both afferent classes. The temporal frequency with which dots cross a single point on the skin is proportional to the scanning velocity and inversely proportional to the dot spacing; at a dot spacing of $2 \mathrm{~mm}$ the scanning velocities 20,60 , and $90 \mathrm{~mm} / \mathrm{sec}$ result in temporal stimulus frequencies of approximately 7,20 , and $30 \mathrm{dots} / \mathrm{sec}$, respectively.

\section{Effect of scanning velocity: FAII and SAII afferents}

The effect of scanning velocity was investigated for four FAII and two SAII afferents. Though not illustrated, the effects of velocity on the responses of these afferent classes were similar to those described for the type I afferents. Thus, although increasing scanning velocity resulted in an increase in the temporal response frequency, the spatial density of action potentials (impulses per dot) was reduced with increasing velocity.

\section{Effect of skin contact force}

The effect of contact force on response was investigated for four FAI fibers and two SAI fibers, using a scanning velocity of 60 
FAll $(n=8)$
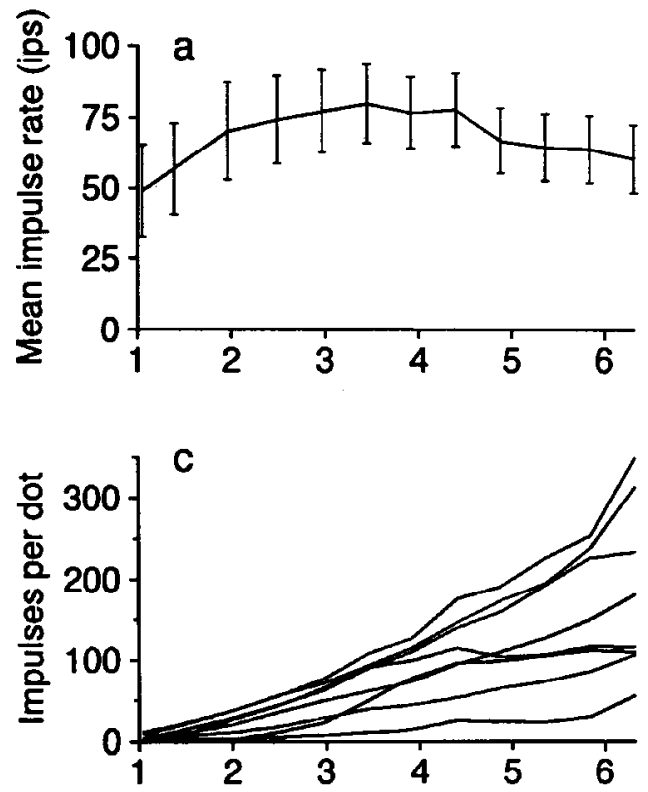

Figure 5. Mean discharge rate and number of impulses evoked per dot versus dot spacing for FAII and SAII fibers. For all data, stimulus scanning velocity was $60 \mathrm{~mm} / \mathrm{sec}$ and application force was 0.6 N. $a$ and $b$, Mean rate ( \pm 1 SE) versus dot spacing for eight FAII and six SAII afferents; for both types mean rate varies little as dot spacing increases. $c$ and $d$, Number of impulses evoked per dot versus dot spacing for each FAII and SAII fiber. $e$ and $f$, Mean number of impulses per dot ( \pm 1 SE) versus dot spacing for data shown in $c$ and $d$. These measures were obtained by averaging within a "moving window" extending across the spatial event plot as described in Materials and Methods.

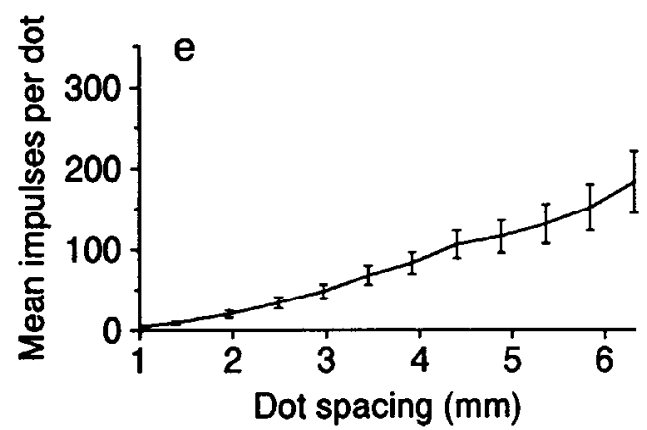

SAll $(n=6)$
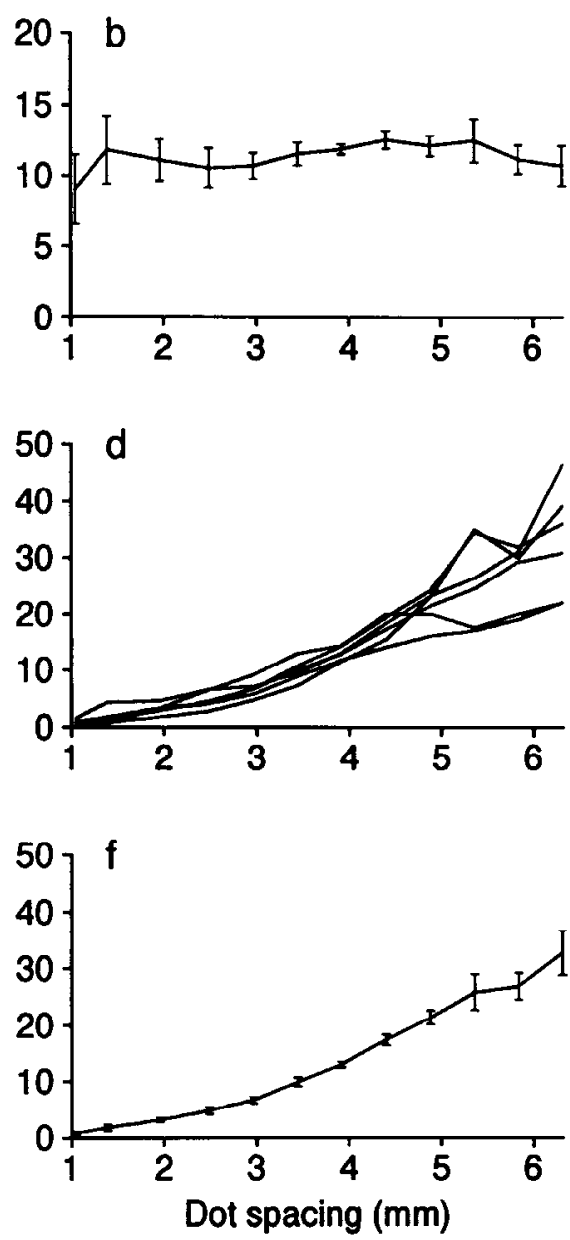

$\mathrm{mm} / \mathrm{sec}$. Figure 8 shows the effect of force for one representative unit of each type. Contact force in the range 0.4-1.0 N has little effect on the responses to the coarse parts of the stimulus pattern (dot spacings greater than 4-5 $\mathrm{mm}$ ). The main effect of force, particularly for the SAI fibers, was an increased response to surface regions with small dot spacings.

\section{Receptive field sensitivity profiles of $S A I$ and $F A I$ afferents}

It was shown earlier (Figs. 2, 3) that for FAI and SAI fibers, the number of impulses evoked per dot remained relatively constant for dot spacings between 5 and $6 \mathrm{~mm}$. Thus, in this range, the responses to each dot were almost unaffected by the presence of neighboring dots and the fibers responded as if each dot were an isolated dot. Under this condition the size of the response cluster associated with each dot is a measure of the size of the fiber's receptive field. In a previous study (Phillips et al., 1990) based on a subset of the data reported here, the receptive field sizes of the $22 \mathrm{FAI}$ and $15 \mathrm{SAI}$ afferents were computed from the linear dimensions of the response clusters associated with individual dots. The sizes of the FAI and SAI fields measured in this way were found to be $6.1 \pm 1.6 \mathrm{~mm}^{2}(n=22)$ and 4.8 $\pm 1.2 \mathrm{~mm}^{2}(n=15)$, respectively (mean $\left.\perp \mathrm{SD}\right)$. The corresponding median values were $5.7 \mathrm{~mm}^{2}$ and $4.9 \mathrm{~mm}^{2}$. The following analysis investigates the profiles of sensitivity within the receptive fields of five FAI and five SAI afferents stimulated at a scanning velocity of $20 \mathrm{~mm} / \mathrm{sec}$. The detailed internal structure of the receptive fields emerged most clearly at this low velocity. Figure $9 a$ shows the impulse pattern produced by a single SAI afferent when a single dot was scanned repeatedly across its receptive field. The responses are limited to a well-defined area, indicating that the border of the field was quite distinct. Although the impulses appear quite evenly distributed, closer analysis shows that there is consistent sweep-to-sweep variation in impulse rate, indicating variations in sensitivity within the receptive field. Figure $9 b$ shows silhouettes of impulse rate plots corresponding to each scan shown in Figure $9 a$. Figure $9 c$ shows a contour plot derived by combining the data in panels $a$ and $b$. The solid contour lines represent isofrequency lines defined on the basis of the instantaneous firing frequencies shown in panel $b$. A number of zones of higher sensitivity arc apparent in the contour plot. Figure $9, d-f$, shows these receptive field characteristics in a different manner. In Figure $9 d$ each action potential is represented by a vertical bar whose height is proportional to the instantaneous response frequency. In Figure 9 , $e$ and $f$, the variability in firing rate within the field is emphasized by representing action potentials as vertical bars with heights proportional to the square and cube of the instantaneous frequency, respectively. The zones of higher sensitivity shown in Figure $9 c$ are also apparent in Figure 9, $e$ and $f$. The fact that these zones reflect variations in sensitivity rather than random 
Dot spacing, $\mathrm{mm}$

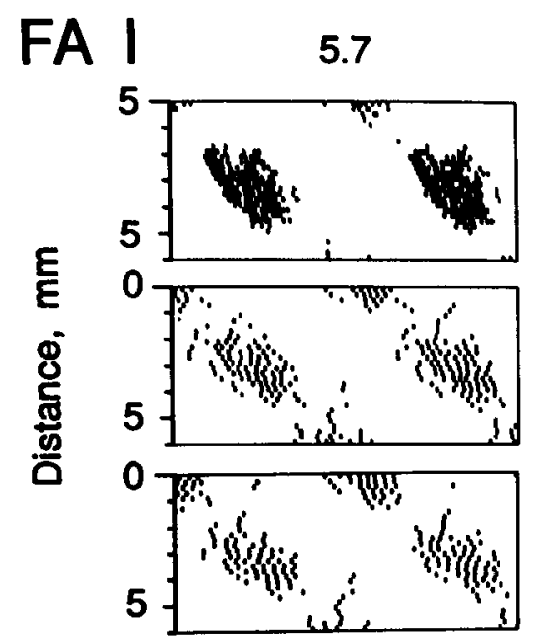

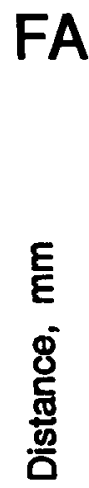

\section{SA I}
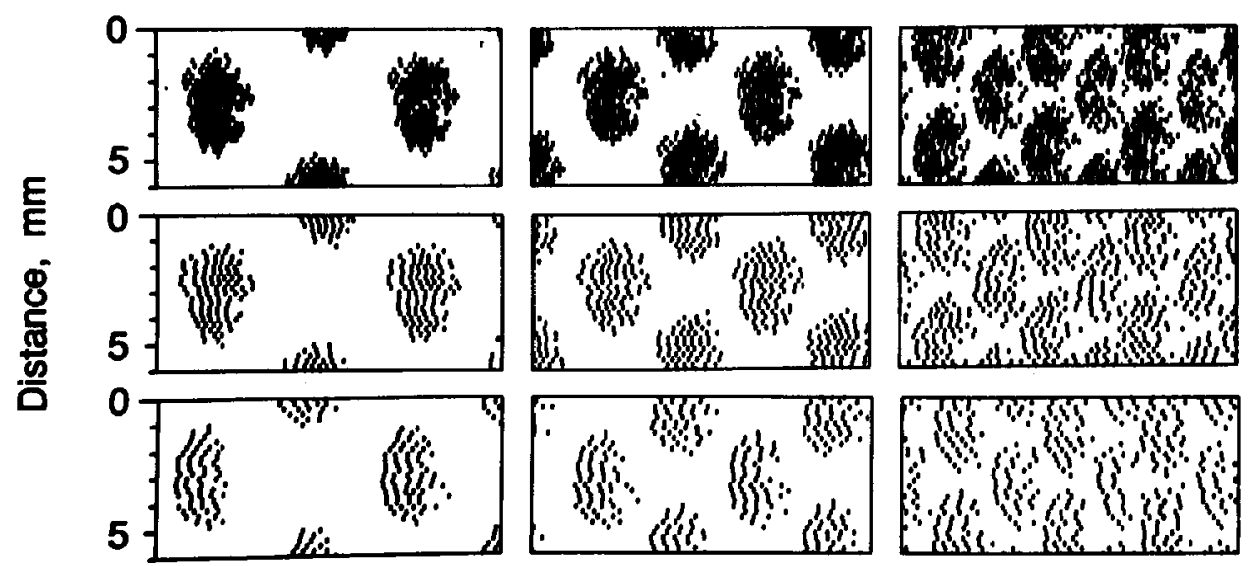

2.8
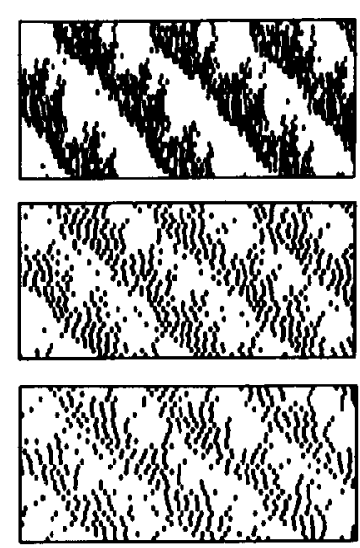

1.7

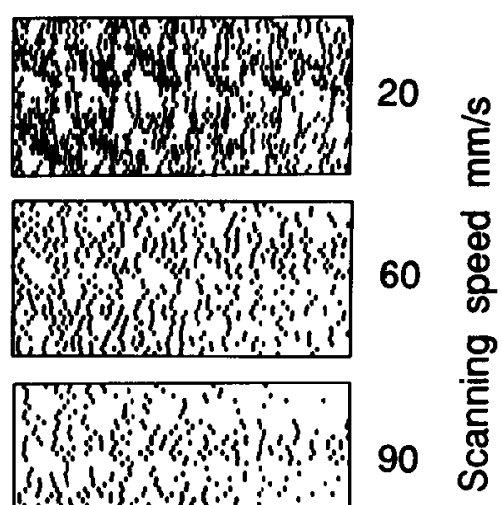

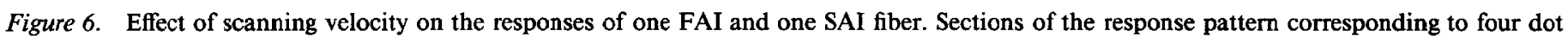
spacings $(1.7,2.8,4.0$, and $5.7 \mathrm{~mm})$ are shown at each of three velocities $(20,60$, and $90 \mathrm{~mm} / \mathrm{sec})$. Application force, $0.6 \mathrm{~N}$.

FAl
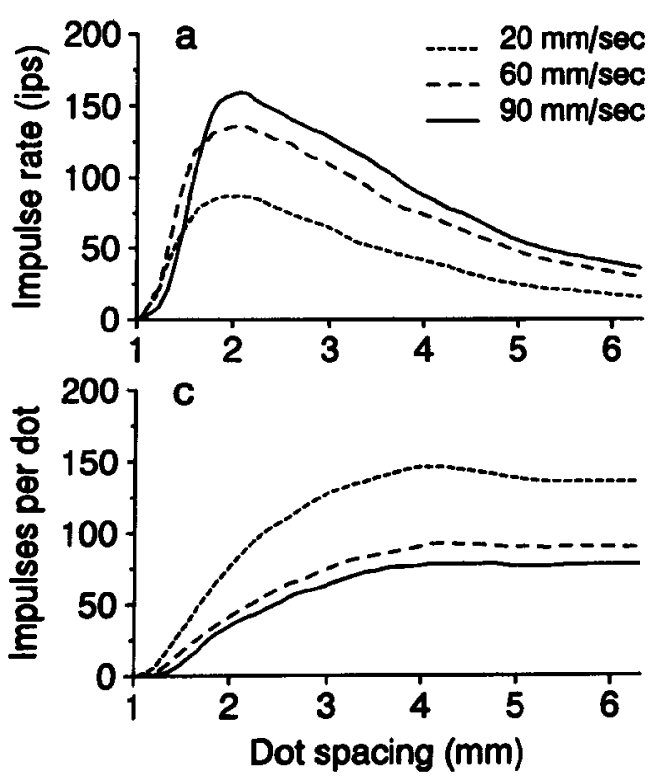

SAI

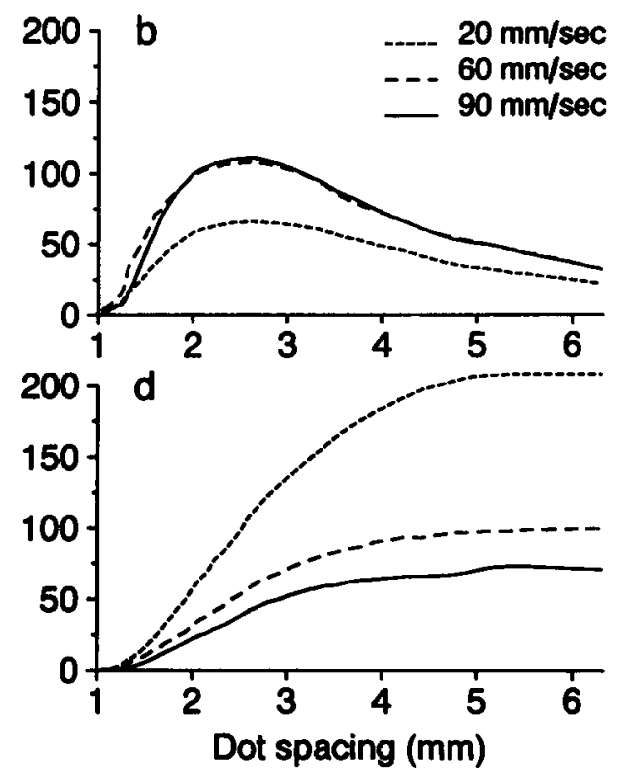

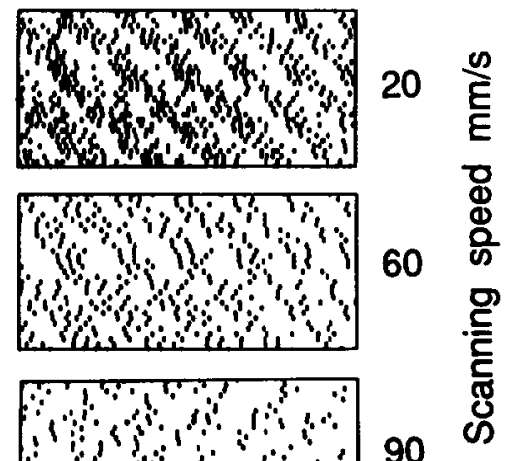

Figure 7. Effect of scanning velocity on mean discharge rate and mean number of impulses per dot versus dot spacing for one FAI and one SAI fiber (same fibers as in Fig. 6). Contact force, 0.6 $\mathrm{N}$. Mean response rate (measured in impulses/sec) generally increases with increasing scanning velocity. However, the number of impulses evoked per dot decreases with increasing scanning velocity. 
Figure 8. Effect of contact force on mean discharge rate and mean number of impulses per dot versus dot spacing for one FAI and one SAI fiber (same fibers as in Fig. 6). Scanning vclocity, $60 \mathrm{~mm} / \mathrm{sec}$ in each case. Impulse rate generally increases with increasing application force (particularly for the SAI afferent at close dot spacing) but the number of impulses evoked per dot is not markedly affected.
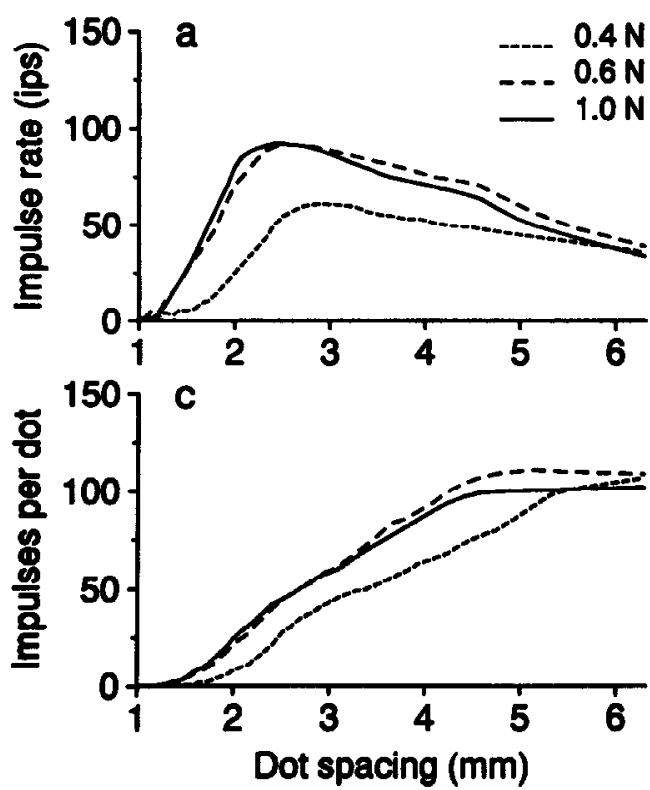
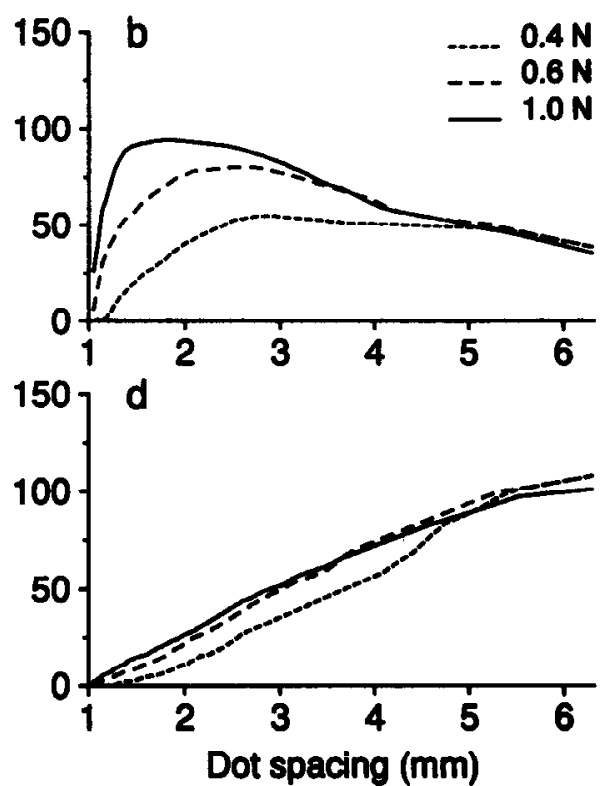

fluctuations in response rate can be seen by comparing the neuron's responses to different dots in the tetragonal pattern. Figure 10 illustrates this repeatability, showing a section of a spatial event plot containing responses to three neighboring dots. All
FAI and SAI fibers analyzed showed multiple zones of high sensitivity within their fields. Figure 11 shows the receptive field topography of four SAI and four FAI afferents plotted in the same way as in Figure $9 f$. The hatched areas indicate zones of
Figure 9. Response of one SAI fiber to an isolated embossed dot. Stimulus scanning velocity, $20 \mathrm{~mm} / \mathrm{sec}$; dot spacing, $5.0 \mathrm{~mm}$. The solid circle (upper left) shows the relative size of the top of the scanning dot. $a$, Simple spatial event plot outlining the receptive field. Each vertical tick represents the occurrence of an action potential. The thin horizontal lines show the consecutive paths of the dot scanning across the reccptive ficld. $b$, Impulse rate silhouettes showing the instantaneous discharge frequencies along each of the scan paths shown in $a$ (the vertical distance between adjacent baselines corresponds to 200 ips). $c$, Receptive field sensitivity profile. Contour lines (isofrequency lines derived from $b$ and superimposed on the spatial event plot shown in $a$ ) were drawn by hand to give the best estimate within the constraints of the action potential spacing. Solid contour lines indicate discharge frequencies of 50,100 , 150 , and 200 ips. Areas enclosed by broken lines indicatc troughs in rcsponse rate. $d$, Spatial event plot (as in a) except with neural events shown as vertical bars whose lengths are proportional to the instantaneous discharge rate (see Materials and Methods). $e$ and $f$, As for $d$ except that the lengths of the bars are proportional to the square (e) and cube $(f)$ of the instantaneous frequency. (For $d-f$, separation between adjacent horizontal lines represents 225 ips, $225 \mathrm{ips}^{2}$, and $225 \mathrm{ips}^{3}$, respectively).

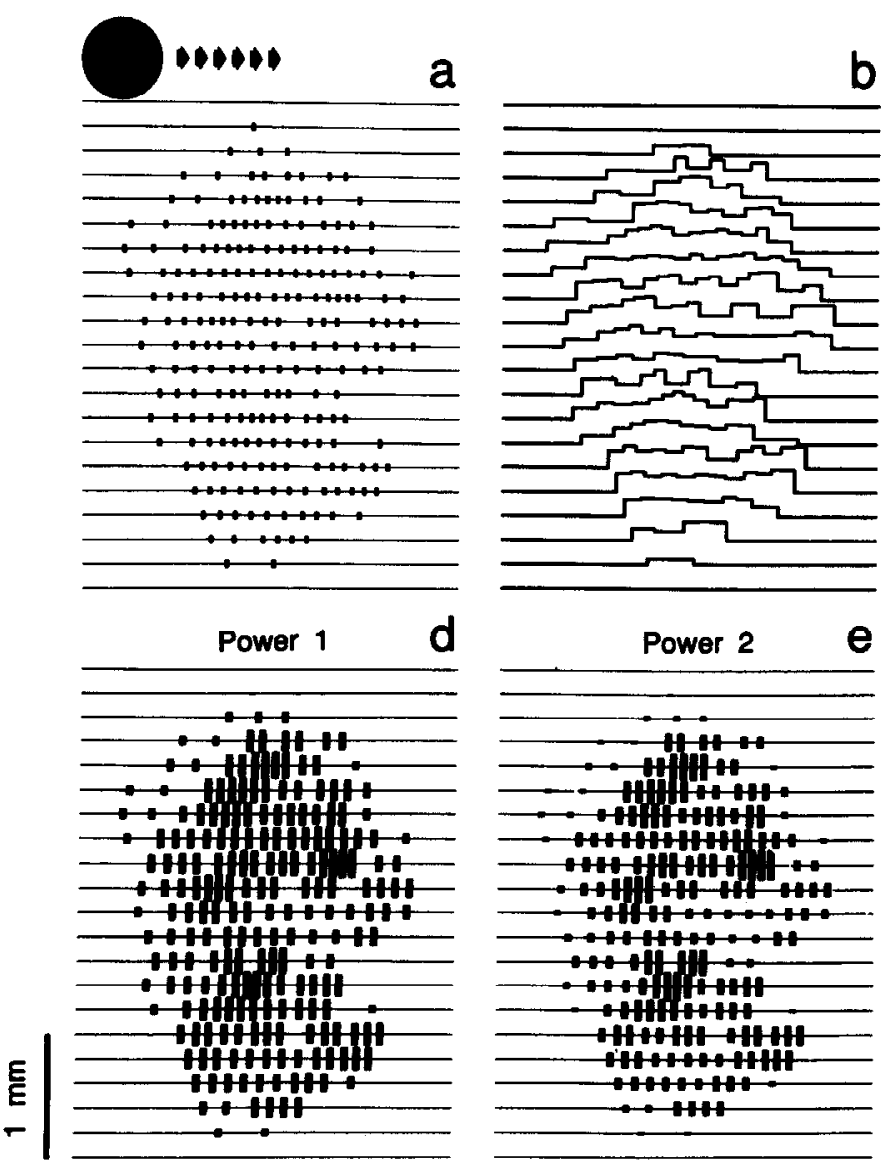

b

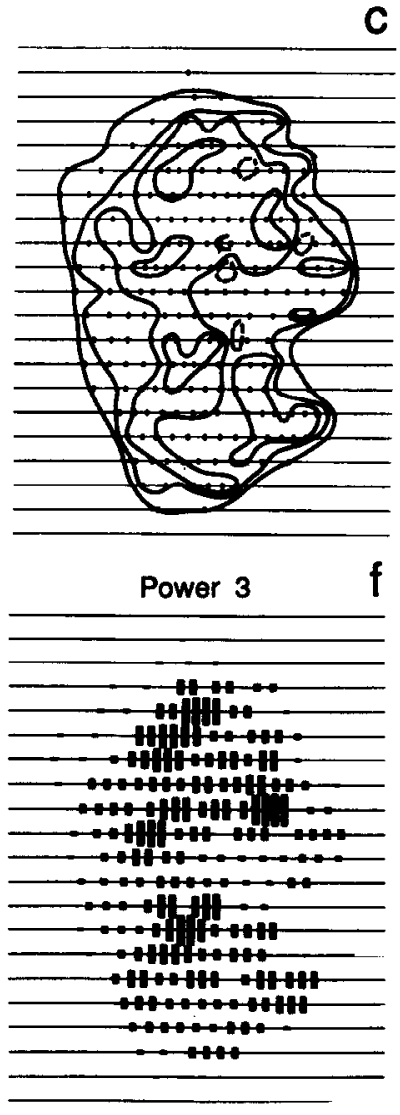



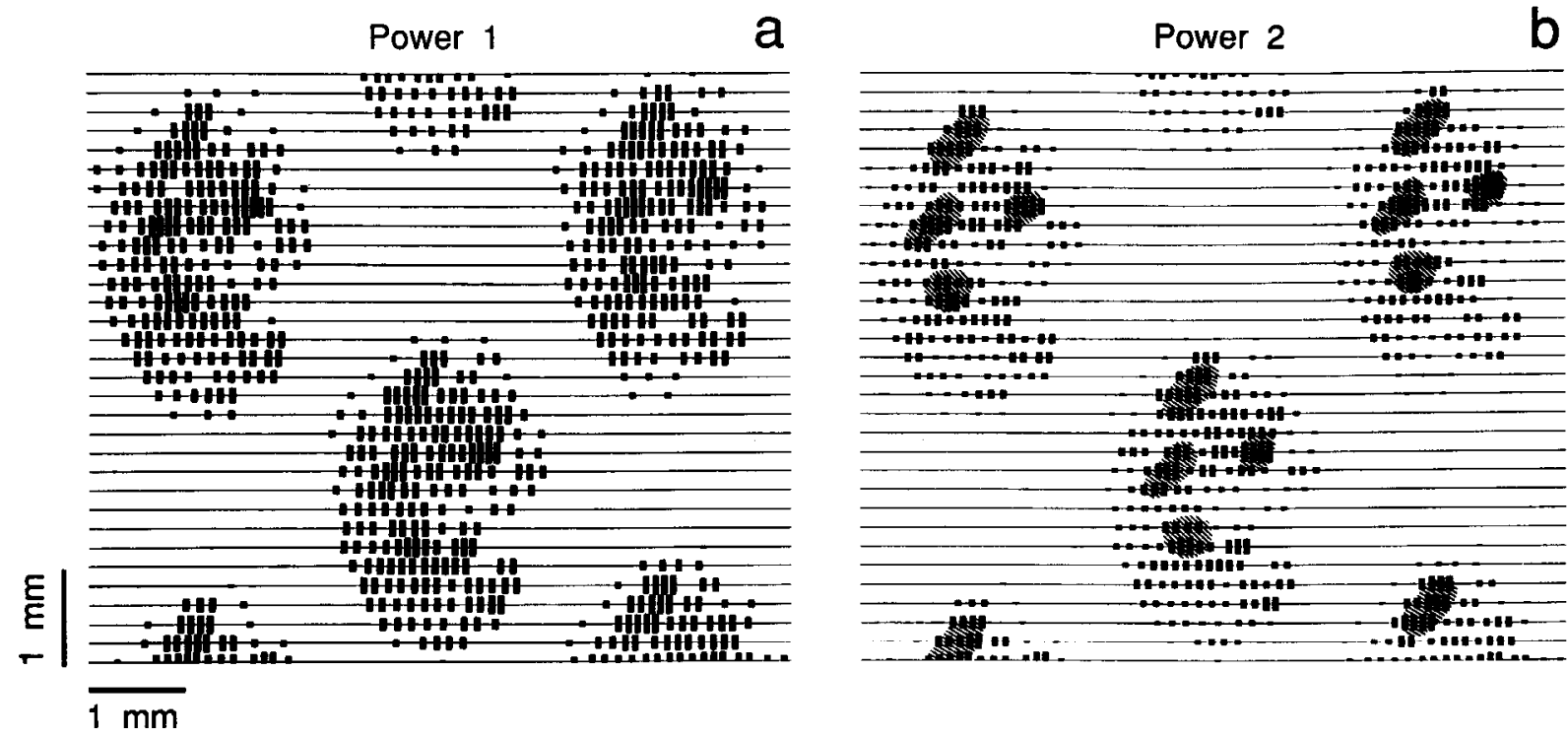

Figure 10. Multiple mapping of the receptive field sensitivity profile of one SAI fiber (same fiber as in Fig. 9) generated by several neighboring raised dots scanning across the field. Dot spacing, $3.5 \mathrm{~mm}$; scanning speed, $20 \mathrm{~mm} / \mathrm{sec}$; application force, $0.6 \mathrm{~N}$. Field profiles were constructed as in Figure 9, $d$ and $e$. The hatched areas outline the zones of highest sensitivity within the field. The repeatability in the positions of these zones within the different representations of the field is apparent. (A bar length corresponding to the vertical distance between adjacent horizontal lines represents 200 ips in $a$ and 200 ips $^{3}$ in $b$.)

highest sensitivity that appeared in the same relative locations within the multiple representations of the fields mapped by neighboring dots. For several afferents, the receptive field sensitivity profiles obtained at different velocities $(20,60$, and 85 $\mathrm{mm} / \mathrm{sec}$ ) were compared. Though not illustrated, the definition of the sensitive zones markedly deteriorated with increasing velocity, though their relative locations within the fields appeared unaltered. Typically, when plotted at $20 \mathrm{~mm} / \mathrm{sec}$, the receptive fields of the five SAI fibers had fewer (three to five) well-defined sensitive zones than the fields of the five FAI fibers (five to eight zones), though the receptive field areas of this subsample were not statistically different [FAI, $7.4 \pm 2.4 \mathrm{~mm}^{2}$; SAI, $6.1 \pm 0.5 \mathrm{~mm}^{2}$ (mean $\pm \mathrm{SD}$ )]. The peak firing rates within the sensitive zones were generally higher for SAI [230-325 impulses/sec (ips)\} than for FAI fibers (140-260 ips). FAII and SAII receptive fields were not analyzed for multiple sensitive zones because even at the widest dot spacings the response clusters from these afferents coalesced, and the boundaries of their large receptive field could not be determined in this way.

\section{Discussion}

Responses of the four classes of human mechanoreceptive afferents were studied by scanning an array of embossed dots with variable dot spacing across their receptive fields. Their spatial resolving capacity was assessed by plotting responses from single afferents in the form of spatial event plots and observing the dot spacing at which spatial structure within the response broke down. FAI and SAI afferents consistently resolved the spatial pattern of the stimulus for dot spacings greater than $1.5 \mathrm{~mm}$. Although the FAII and SAII afferents responded to the stimulus pattern at all dot spacings, they only resolved individual dots at much wider spacings $(3-4 \mathrm{~mm})$. The study of monkey afferents by Connor et al. (1990) is similar to this study in that tetragonal patterns of dots were used as stimuli, with the same dot spacing and dot diameter as in this study. However, the dot height was quite different ( $350 \mu \mathrm{m}$ vs. $500 \mu \mathrm{m}$ in this study). Because of the difference in height, the dots used in this study felt sharper and the dot patterns felt rougher at all dot spacings. Despite this difference, the responses of human SAI, FAI, and FAII afferents were similar to those observed for monkey SAI, RA, and PC afferents. Thus, the primary difference between monkey and human peripheral tactile mechanisms appears to be the lack of SAII afferent fibers innervating the distal pads of monkeys. Neither SAII afferents nor spatial event plots like those displayed for human SAII fibers in this study (and by Phillips et al., 1990) have been reported for the glabrous skin of the monkey fingerpads.

One straightforward characterization of a fiber's response to the scanning pattern was the mean impulse rate associated with different parts of the pattern (i.e., to different dot spacings). The mean response rate of SAI and FAI afferents was low for widely spaced dots, progressively increased as dot spacing decreased to peak at $2-3 \mathrm{~mm}$ dot spacing, and then fell sharply at finer dot spacings. In contrast, the mean response rate of FAII afferents versus dot spacing varied markedly across fibers and their responses could not easily be characterized. The mean response rate of SAII afferents was largely unaffected by dot spacing. In fact, SAII responses are qualitatively and quantitatively different from those of the other three classes. Whereas the dot patterns evoked similar impulse rates in SAI, FAI, and FAII afferents, the rates evoked in SAII afferents were much lower, forming a distinct class based on this measure alone. Using the peak impulse rate evoked by the pattern scanning at $60 \mathrm{~mm} /$ $\mathrm{sec}$ as a measure, the minimum, median, and maximum values were 30, 96, and 162 ips for FAIs; 27, 95, and 134 ips for FAIIs; 37, 73, and 109 ips for SAIs; but only 11, 13, and 17 ips for SAII affercnts. Moreover, intraneural microstimulation studies of single afferents in humans have shown that activation of single FAI, FAII, or SAI afferents can evoke conscious sensations, whereas activation of single SAII afferents generally evokes no 


\section{SA I}

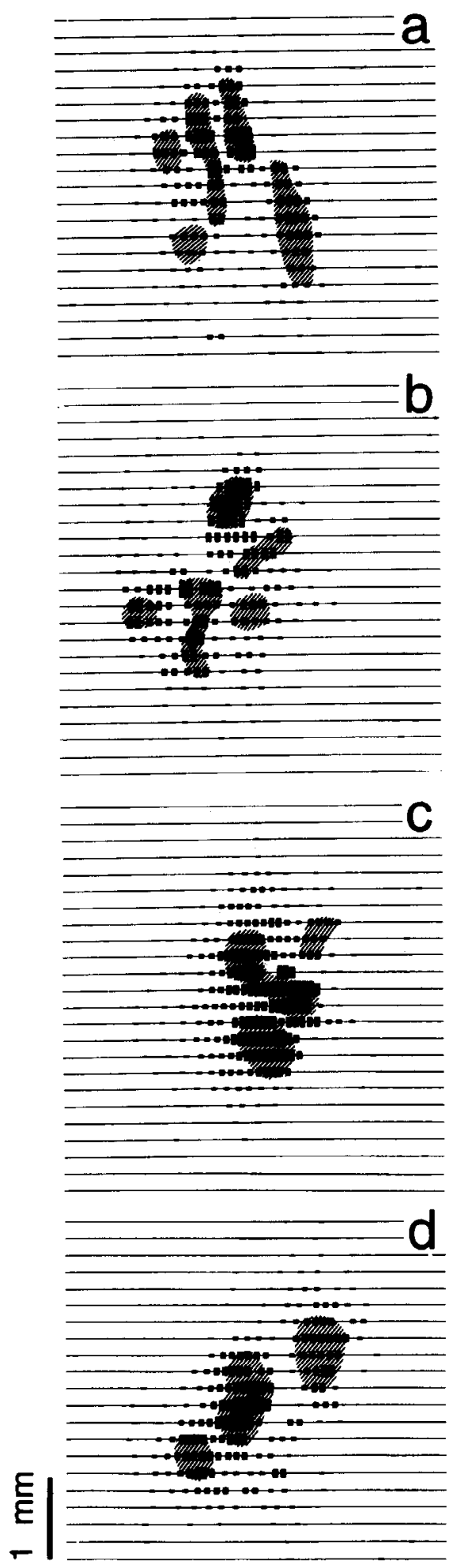

FA I

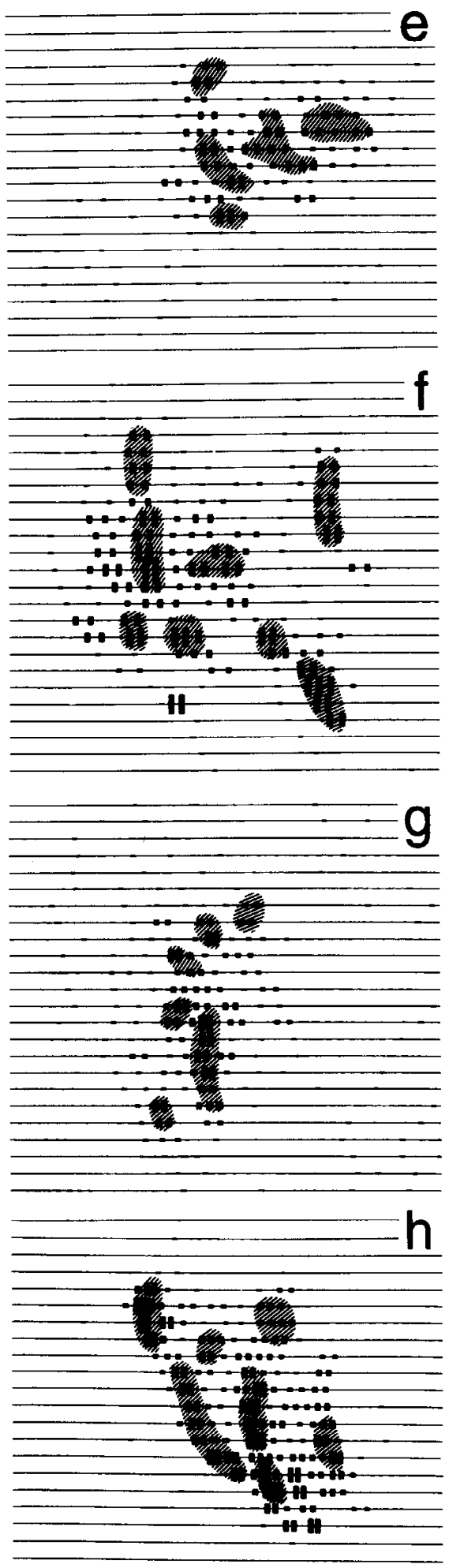

Figure 11. Receptive field sensitivity profiles of four SAI afferents and four FAI afferents. Spatial event plots show neural events as vertical bars whose lengths are proportional to the cube of the instantaneous discharge rate. The hatched areas show the zones of highest sensitivity that appeared in the same relative position within the multiple representations of each field provided by neighboring dots (not shown), but as illustrated in Figure 10. Scanning velocity, $20 \mathrm{~mm} / \mathrm{sec}$; application force, 0.6 $\mathrm{N}$. Bar equal to the distance between contour lines: $a, 250$ ips $^{3} ; b, g$, and $h$, $275 \mathrm{ips}^{3} ; c, 300 \mathrm{ips}^{3} ; d, 325 \mathrm{ips}^{3} ; e, 175$ ips $^{3} ; f, 200$ ips $^{3}$. sensation (Ochoa and Torebjork, 1983). This evidence, combined with the poor resolving power of individual SAII afferents, makes it unlikely that their responses contribute to tactile spatial resolution, and their role remains unclear.

Varying scanning velocity between 20 and $90 \mathrm{~mm} / \mathrm{sec}$ had little effect on the clarity with which spatial detail was repre- sented in the SEPs reconstructed from responses of all four afferent classes. Although impulse rate increased with increasing velocity, the number of impulses evoked per dot decreased with increasing velocity as has been observed for monkey afferents (Darian-Smith et al., 1980; Johnson and Lamb, 1981; Connor et al., 1990). Varying contact force between 0.3 and $1.0 \mathrm{~N}$ also 


\section{A Dot spacing ca $3.6 \mathrm{~mm}$}

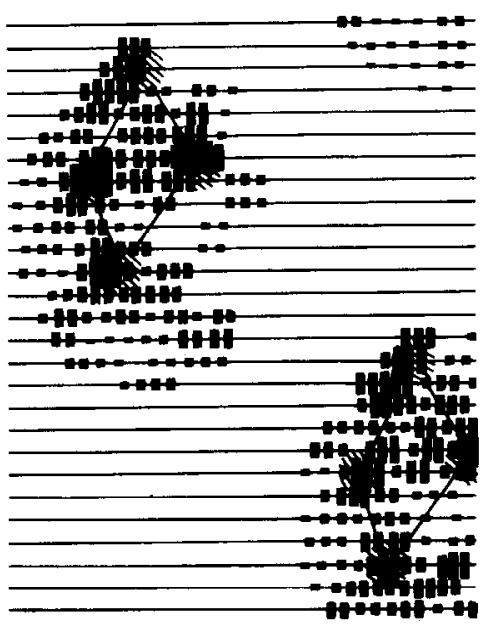

\section{Dot spacing ca $1.4 \mathrm{~mm}$}

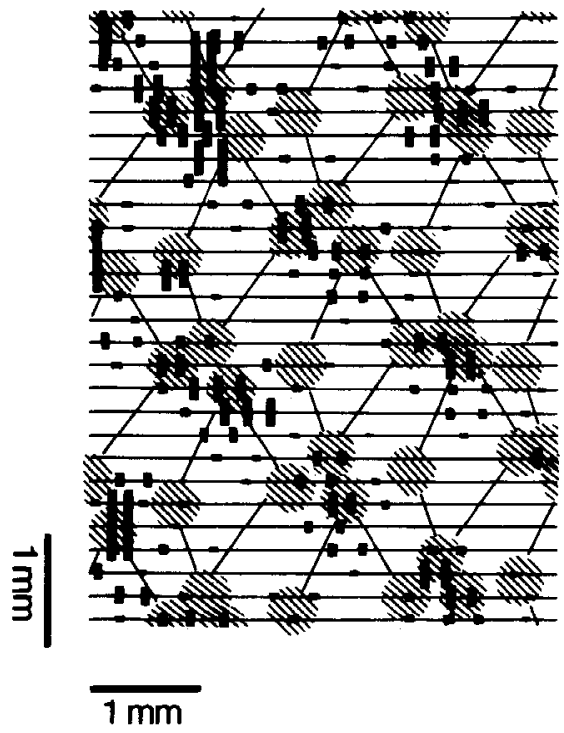

B Dot spacing ca $1.8 \mathrm{~mm}$

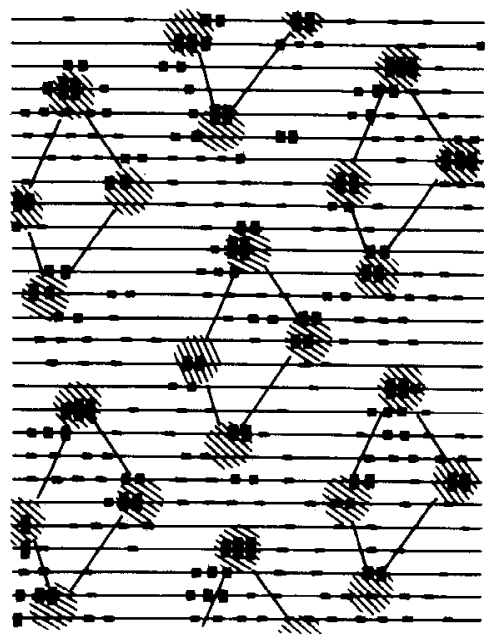

D Dot spacing ca $1.2 \mathrm{~mm}$

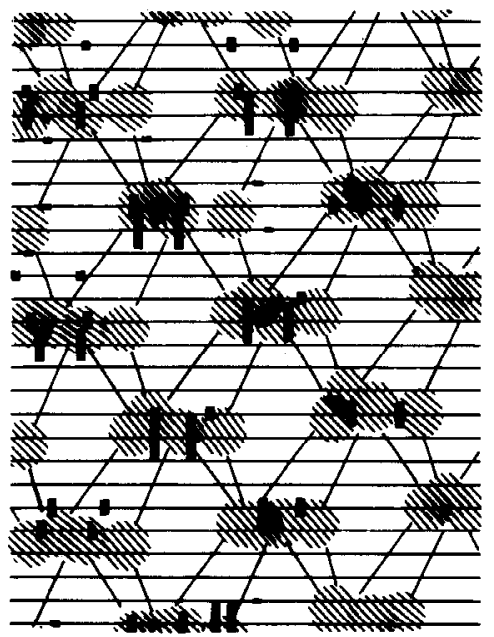

Figure 12. Interaction of receptive field structure with closely spaced dots. $A-D$ show subsections of one spatial event plot at four different dot spacings. The positions of the four zones of highest sensitivity within the field (hatched zones joined by straight lines) were determined from the responses to widely spaced dots. With decreasing dot spacing the relative positions of the shaded areas were determined by manually superimposing a scaled template of the stimulus pattern over the response pattern. It is apparent that as dot spacing decreases the effective size of the receptive field is reduced (compare the large overall responsive area in $A$ with the smaller responsive areas in $B$ ). In $B$, responses only occur when dots pass over the four most sensitive zones. With further reduction in dot spacing ( $C$ and $D$ ) responses occur when sensitive zones overlap. The overlap of sensitive zones indicates that these zones were simultaneously stimulated by different dots. Scanning velocity, $20 \mathrm{~mm} / \mathrm{sec}$; application force, $0.6 \mathrm{~N}$. The vertical distance between two adjacent horizontal lines: $A$ and $B, 200$ ips $^{3} ; C, 100$ ips $^{3} ; D, 50$ ips $^{3}$.

had little effect on the representation of spatial detail in the SEPs reconstructed from FAI or SAI responses except at the finer dot spacings. Again, these effects are similar to those seen in monkey (Johnson and Lamb, 1981).

At coarse dot spacings (6 $\mathrm{mm}$ to about $4 \mathrm{~mm}$ spacing) SAI and FAI afferents responded to each dot with minimal effects from neighboring dots. The size of the response cluster in the SEP associated with each dot was taken as a measure of the size of the receptive field of the fiber (Johnson and Lamb, 1981). The mean receptive field areas for FAI and SAI afferents were 6.1 and $4.8 \mathrm{~mm}^{2}$, respectively. These areas are slightly smaller than the receptive fields of human afferents measured with punctate stimuli delivered manually using von Frey hairs (Johansson and Vallbo, 1980). Areas of RA and SA fields in monkey measured using scanned dot arrays are comparable (RA, $11.6 \mathrm{~mm}^{2}$; $\mathrm{SA}, 4.5 \mathrm{~mm}^{2}$; Johnson and Lamb, 1981). The data from both monkey and human imply that the SAI fields are somewhat smaller than FAI fields. However, identifiable tetragonal structure was still visible in the responses of the FAI and SAI afferents at dot spacings that were considerably smaller than the overall receptive field dimensions. Thus, overall receptive field size was not a reliable predictor of the spatial resolving capacity of these afferents.

Analysis of the instantaneous response rates within the response clusters associated with widely spaced dots showed that the receptive fields of FAI and SAI afferents were made up of a number of small zones of high sensitivity. Similar receptive field topography has been reported for human FAI and SAI afferents using punctate mapping techniques (Johansson, 1978) and for monkey afferents using scanned dots (Johnson and Lamb, 1981) and stationary gratings (Phillips and Johnson, 1981a,b). The interpretation has been that the separate zones of maximal sensitivity within FAI fields correspond to the different spatial locations of a number of Meissner's corpuscles connected to a single axon while the zones within SAI ficlds correspond to the different spatial locations of a number of Merkel cell complexes innervated by a single axon (see Johansson, 1978). Because the stimulus used in this study included a number of widely spaced 
dots, the receptive field structure of each afferent was mapped out many times, allowing the relative positions of the sensitive zones to be reliably determined. In this study FAI afferents typically had five to eight well-defined scnsitive zones, whereas SAI afferents typically had three to five such zones. There is anatomical evidence of ample branching of large-diameter axons innervating the Meissner and Merkel mechanoreceptors of human glabrous skin. Each axon leaving the digital nerve branches at least three to four times before reaching the papillary layer of the dermis (Cauna, 1956, 1959), suggesting that each axon innervates a minimum of 8-16 terminals. Thus, the number of highly sensitive zones found in the present study is lower than might be expected. One reason for this may be that the diameter of the scanning dots used in this study was too large to resolve all the individual sensitive zones associated with each receptive field. [The $0.7-\mathrm{mm}$-diameter dots were larger than the width of a single papillary ridge (about $0.5 \mathrm{~mm}$ ) and much larger than the distance separating the ridges (about $0.25 \mathrm{~mm}$ ).] Johansson (1978), using 0.5-mm-diameter probes with hemispherical tips applied with forces close to threshold, reported 12-17 sensitive zones within each FAI field.

As dot spacing was reduced below about $4 \mathrm{~mm}, \mathrm{FAI}$ and SAI responses to each dot (number of impulses per dot) progressively decreased (Fig. 3). Similar interactions between adjacent elements of a spatial pattern resulting in a reduction of response have been observed in monkey primary afferents for scanning gratings and dots (Johnson and Lamb, 1981; Goodwin and Morley, 1987a; Sathian et al., 1989) and for vertically indented gratings with no lateral movement (Phillips and Johnson, 1981a). It has been shown that for vertically indented gratings, the reduction in response in monkey SA afferents due to the proximity of neighboring elements of a pattern can be quantitatively accounted for by a reduction in the effective stimulus (compressive strain) at the receptor terminal (Phillips and Johnson, 1981b). The compressive strain at the receptor is reduced by the presence of adjacent stimulus elements because the skin loads are distributed amongst more elements. It seems likely that a similar mechanism acts to reduce response to closely spaced elements of a scanning stimulus, though the identity of the effective (dynamic) stimulus at the receptor terminal of FAI and SAI afferents during scanned stimuli has not been identified. Despite the depression of the response to a single dot by the presence of neighboring dots, the principal features of the receptive field sensitivity profiles could be traced as dot spacing decreased. In Figure 12 (same SAI unit as in Figs. 9, 10), the four most sensitive zones within the field (indicated by hatched circles) have been joined with straight lines. As dot spacing decreases, the areal extent of the response clusters associated with each dot decrease, as can be seen by comparing the response clusters in Figure $12 \mathrm{~A}$ with those in Figure $12 \mathrm{~B}$. That is, the effective size of the receptive field is reduced as dot spacing is reduced. As dot spacing is reduced further, responses associated with onc or more highly sensitive zones often disappear (see right part of Fig. 12C). However, as was described earlier (and can be seen in Fig. 12C), there was generally a visible patterning of responses as long as the unit remained firing. Inspection of the response patterns of the five FAI and SAI fibers to very closely spaced dots showed that clusters of action potentials tended to occur within the SEPs at locations where two or more sensitive zones were simultaneously stimulated by different dots. This is seen most clearly for the responses illustrated in Figure 12, $C$ and $D$ : the action potentials are clustered in regions where sensitive zones overlap or occur close together. Action potentials are absent in regions where sensitive zones occur in isolation. Thus, it appears that when the dots in the stimulus pattern spatially coincidc with a subsct of sensitive zones then the afferent unit may be activated by superposition of the effects of different dots acting on different sensitive zones within the same receptive field. We believe that this phenomenon of "spatial coincidence" between the stimulus pattern and the receptive field topography might account for the presence of structured responses in the SEPs at dot spacings that are much smaller than the overall dimensions of the receptive field. Such spatial summation of the effects of stimulating more than one terminal within the receptive field of a single axon might imply that the threshold for spike initiation is set at the branch point of the parent axon rather than within the terminals themselves.

\section{References}

Cauna N (1956) Nerve supply and nerve endings in Meissner's corpuscles. Am J Anat 99:315-350.

Cauna $N$ (1959) The mode of termination of the sensory nerves and its significance. J Comp Neurol 113:169-199.

Connor CE, Hsiao SS, Phillips JR, Johnson KO (1990) Tactile roughness: neural codes that account for psychophysical magnitude estimates. J Neurosci 10:3823-3836.

Darian-Smith I, Oke LE (1980) Peripheral neural representation of the spatial frequency of a grating moving across the monkey's fingerpad. J Physiol (Lond) 309:117-133.

Darian-Smith I, Davidson I, Johnson KO (1980) Peripheral neural representation of spatial dimensions of a textured surface moving across the monkey's finger-pad. J Physiol (Lond) 309:135-146.

Goodwin AW, Morley JW (1987a) Sinusoidal movement of a grating across the monkey's fingerpad: representation of grating and movement features in afferent fiber responses. J Neurosci 7:2168-2180.

Goodwin AW, Morley JW (1987b) Sinusoidal movement of a grating across the monkey's fingerpad: effect of contact angle and force of the grating on afferent fiber responses. J Neurosci 7:2192-2202.

Goodwin AW, John KT, Sathian K, Darian-Smith I (1989) Spatial and temporal factors determining afferent fiber responses to a grating moving sinusoidally over the monkey's fingerpad. J Neurosci 9:12801293.

Gordon I, Cooper C (1975) Improving one's touch. Nature 256:203204.

Johansson RS (1978) Tactile sensibility of the human hand: receptive field characteristics of mechanoreceptive units in the glabrous skin area. J Physiol (Lond) 281:101-123.

Johansson RS, LaMotte RH (1983) Tactile detection thresholds for a single asperity on an otherwise smooth surface. Somatosens Res 1: 21-31.

Johansson RS, Vallbo AB (1979) Tactile sensibility in the human hand: relative and absolute densities of four types of mechanoreceptive units in the glabrous skin. J Physiol (Lond) 286:283-300.

Johansson RS, Vallbo AB (1980) Spatial properties of the population of mechanoreceptive units in the glabrous skin of the human hand. Brain Res 184:353-366.

Johansson RS, Landstrom U, Lundstrom R (1982) Sensitivity to edges of mechanoreceptive afferent units innervating the glabrous skin of the human hand. Brain Res 244:27-32.

Johnson KO, Lamb GD (1981) Neural mechanisms of spatial tactile discrimination: neural patterns evoked by Braille-like dot patterns in the monkey. J Physiol (Lond) 310:117-144.

Johnson KO, Phillips JR (1984) Spatial and non-spatial neural mechanisms underlying tactile spatial discrimination. In: Wenner-Gren Center international symposium series, Vol 41 , Somatosensory mechanisms (von Euler C, Franzen O, Lindblom U, Ottoson D, eds), pp 237-248. London: Macmillan.

Johnson KO, Phillips JR (1988) A rotating drum stimulator for scanning embossed patterns and textures across the skin. J Neurosci Methods 22:221-231.

Katz D (1925) Der Aufbau der Tastwelt. Reprint; orig publ in Z Psychol, Vol 11. In: The world of touch (Krueger LE, transl), pp 7589. Hillsdale, NJ: Erlbaum, 1989. 
Knibestol M, Vallbo AB (1970) Single unit analysis of mechanoreceptor activity from the human glabrous skin. Acta Physiol Scand 80:178-195.

Lamb GD (1983a) Tactile discrimination of textured surfaces: psychophysical performance measurements in humans. J Physiol (Lond) 338:551-565.

Lamb GD (1983b) Tactile discrimination of textured surfaces: peripheral neural coding in the monkey. J Physiol (Lond) 338:567-587.

LaMotte RH, Srinivasan MA (1987a) Tactile discrimination of shape: responses of slowly adapting mechanoreceptive afferents to a step stroked across the monkey fingerpad. J Neurosci 7:1655-1671.

LaMotte RH, Srinivasan MA (1987b) Tactile discrimination of shape: responses of rapidly adapting mechanoreceptive afferents to a step stroked across the monkey fingerpad. J Neurosci 7:1672-1681.

LaMotte RH, Whitehouse J (1986) Tactile detection of a dot on a smooth surface: peripheral neural events. J Neurophysiol 56:11091128.

Lederman SJ (1974) Tactile roughness of grooved surfaces: the touching process and effects of macro- and microsurface structure. Percept Psychophysiol 16:385-395.

Morley JW, Goodwin AW (1987) Sinusoidal movement of a grating across the monkey's fingerpad: temporal patterns of afferent fiber responses. J Neurosci 7:2181-2191.

Morley JW, Goodwin AW, Darian-Smith I (1983) Tactile discrimination of gratings. Exp Brain Res 49:291-299.

Ochoa J, Torebjork E (1983) Sensations evoked by intraneural microstimulation of singlc mcchanoreceptor units innervating the human hand. J Physiol (Lond) 342:633-654.
Phillips JR, Johnson KO (1981a) Tactile spatial resolution. II: Neural representation of bars, edges and gratings in monkey primary afferents. J Neurophysiol 46:1192-1203.

Phillips JR, Johnson KO (1981b) Tactile spatial resolution. III: A continuum mechanics model of skin predicting mechanoreceptor responses to bars, edges, and gratings. J Neurophysiol 46:1204-1225.

Phillips JR, Johansson RS, Johnson KO (1990) Representation of Braille characters in human nerve fibres. Exp Brain Res 81:589-592.

Sathian K, Goodwin AW, John KT, Darian-Smith I (1989) Perceived roughness of a grating: correlation with responses of mechanoreceptive afferents innervating the monkey's fingerpad. J Neurosci 9:12731279.

Srinivasan MA, LaMotte RH (1987) Tactile discrimination of shape: responses of slowly and rapidly adapting mechanoreceptive afferents to a step indented into the monkey fingerpad. J Neurosci 7:16821697.

Talbot WH, Darian-Smith I, Kornhuber HH, Mountcastle VB (1968) The sense of flutter-vibration: comparison of the human capacity with response patterns of mechanoreceptive afferents from the monkey hand. J Neurophysiol 31:301-334.

Vallbo AB, Hagbarth KE (1968) Activity from skin mechanoreceptors recorded percutaneously in awake human subjects. Exp Neurol 21 270-289.

Vallbo AB, Johansson RS (1984) Properties of cutaneous mechanoreceptors in the human hand related to touch sensation. Hum Neurobiol 3:3-14. 